\title{
PRODUÇÃO E MANEJO DE RESÍDUOS SÓLIDOS DE SAÚDE NO HOSPITAL VETERINÁRIO DA UFPR
}

\section{(Production and Management of Solid Health Service Waste in the Veterinary Hospital at UFPR)}

\author{
ROEDER-FERRARI, L. D. ${ }^{1}$; ANDRIGUETTO FILHO, J. M. ${ }^{2}$; FERRARI, M. V. ${ }^{3}$ \\ ${ }^{1}$ Médica veterinária autonoma \\ ${ }^{2}$ Depto. Zootecnia - UFPR \\ ${ }^{3}$ Depto. Medicina Veterinária - UFPR
}

\begin{abstract}
RESUMO - Os resíduos de serviços de saúde (RSS) são aqueles gerados em todos os serviços relacionados com o atendimento à saúde humana ou animal, e podem ser provenientes de laboratórios de análises clínicas e patológicas, drogarias e farmácias, estabelecimentos de ensino e pesquisa na área da saúde, centros de controle de zoonoses, clínicas, consultórios e hospitais veterinários, dentre outros similares. Neste estudo foi efetuada uma análise da produção dos resíduos do Hospital Veterinário da Universidade Federal do Paraná, assim como um levantamento de dados referentes ao manejo desses resíduos. Verificou-se que as etapas de manejo interno dos RSS não estavam em conformidade com a legislação vigente, e que os manipuladores desconheciam os requisitos mínimos para o gerenciamento de tais resíduos. Caso o hospital adotasse a classificação e segregação conforme determina a Agência Nacional de Vigilância Sanitária (ANVISA), em detrimento da segregação atual, poderia haver uma diminuição de $58 \%$ dos resíduos segregados como RSS.
\end{abstract}

Palavras-chave: Resíduos de serviços de saúde; resíduos veterinários; manejo de resíduos.

ABSTRACT - Health service wastes (RSS) means any waste which is generated in the diagnosis, treatment, or immunization of human beings or animals, in research, or in the production of testing biological, including veterinary clinics and hospitals. In this study, an evaluation of heath service waste generation and management was carried out at the Veterinary Hospital of Federal University of Parana (UFPR). According to the results, it has been verified that the steps in the internal management of the RSS did not agree with the valid legislative guidelines, and the handlers of these wastes had not the knowledge of the minimal guidance to manage it. If the hospital adopted the protocol of the Agencia Nacional de Vigilancia Sanitaria (ANVISA) for classification and segregation of RSS, $58 \%$ of his RSS may be handled as general solid waste and did not require special handling or treatment.

Keywords: health service wastes; veterinary wastes; waste management.

\section{INTRODUÇÃO}

Os resíduos de serviços de saúde (RSS) são aqueles gerados em todos os serviços relacionados com o atendimento à saúde humana ou animal, e podem ser provenientes de laboratórios de análises clínicas e patológicas, drogarias e farmácias, estabelecimentos de ensino e pesquisa na área da saúde, centros de controle de zoonoses, clínicas, consultórios e hospitais veterinários, dentre outros similares (ANVISA, 2004; CONAMA, 2005).

A Resolução da Diretoria Colegiada (RDC) n 306 da ANVISA (Agência Nacional de Vigilância Sanitária), que entrou em vigor em dezembro de 2004, determinou que todos os estabelecimentos de saúde devem elaborar um Plano de Gerenciamento dos RSS e para tanto, o conhecimento da produção e da forma de manejo é indispensável. Anteriormente à publicação desta resolução, o estabelecimento gerador não era responsável pelos RSS produzidos, fato este que colaborou para a falta de controle ou gerenciamento dos próprios resíduos, por parte dos responsáveis da maioria das clínicas e hospitais veterinários do país.

Durante o período de revisão de bibliografia, apenas sete trabalhos sobre resíduos resultantes de atividades clínicas na Medicina Veterinária foram encontrados e, entre eles, apenas um citava os resíduos gerados em clínicas de pequenos animais (KRAUSS, 2003). Na avaliação do conteúdo do mesmo, pôde-se perceber que o estudo havia como objetivo inserir o leitor na problemática dos resíduos gerados na atividade veterinária e não entrava em aspectos práticos relacionados a análises quantitativas ou qualitativas desses resíduos, ou mesmo 
em requisitos mínimos para o seu gerenciamento. Nenhum estudo sobre microbiologia de resíduos provenientes de ambientes veterinários foi encontrado, e portanto estão referenciados trabalhos efetuados em hospitais humanos.

VOLKOW (2003) coletou as seringas utilizadas durante uma semana em um hospital mexicano e afirmou que de 7157 seringas plásticas descartáveis, $5257(73 \%)$ foram utilizadas exclusivamente para diluir medicamentos e administrá-los mediante tubos de terapia intravenosa, ou seja, não tiveram contato com sangue ou outros fluidos corpóreos. O autor salientou que todas as seringas foram classificadas como resíduo infectante, de acordo com norma local.

ANDRADE (1997) efetuou análise microbiológica de RSS em 93 estabelecimentos de saúde e concluiu que a maioria dos microrganismos encontrados nos RSS pesquisados pertence à microbiota normal humana, portanto são incapazes, por si próprios, de causarem uma infecção. Já SUBERKROPP e KLUG (1974), citado por ARMOND e AMARAL, 2001) verificaram a presença nos resíduos domiciliares de microrganismos como Mycobacterium tuberculosis, que apresentou um tempo de sobrevivência de até 180 dias na massa de resíduos e Leptospira interrogans, que sobreviveu até 43 dias. $O$ fato de não existirem estudos semelhantes em ambientes veterinários gera dúvidas quanto à presença e persistência nos resíduos de alguns microrganismos exclusivos de animais, ou mesmo daqueles com potencial zoonótico.

O enquadramento equivocado de um resíduo na categoria perigoso, pode trazer como conseqüência custos elevados para o seu gerenciamento, com a utilização de recursos que, numa sociedade onde os mesmos são escassos e as prioridades muitas, poderiam ser melhor aproveitados (FERREIRA, 1995).

O objetivo deste trabalho foi o diagnóstico qualitativo e quantitativo da geração, manejo e natureza dos RSS do Hospital Veterinário da UFPR, assim como revisão de literatura e legislação vigente.

\section{Material e Métodos}

Entre março de 2004 e setembro de 2005 foi efetuado o levantamento dos RSS com acompanhamento da produção e do manejo interno dos resíduos sólidos gerados no hospital, através de visitas, cumprindo uma jornada média de 90 horas por mês de observação. Foram também efetuadas entrevistas com diversos envolvidos na geração e manipulação dos RSS. Para obtenção de dados quantitativos, procedeu-se a pesagem de todos os resíduos sólidos gerados e segregados pelos manipuladores da seguinte forma: sacos pretos com resíduos classificados como comuns, sacos brancos com resíduos classificados como RSS, caixas contendo resíduos perfuro-cortantes, e carcaças e/ou cadáveres animais dispostos em câmara fria.

PESAGEM 1 - Durante 21 dias de atividades hospitalares, ou seja, sem considerar finais de semana e feriados, entre os dias 25/10/04 e 25/11/04, todos os sacos foram pesados individualmente. Durante este período foi acompanhada a disposição de carcaças ou cadáveres na câmara fria.

PESAGEM 2 - As caixas contendo resíduos perfurocortantes, quando atingiram sua capacidade total, foram pesadas individualmente, durante 0 período de 03/01/05 até 25/08/2005.

PESAGEM 3 - Durante 21 dias, entre 04/05/05 e $02 / 06 / 05$, foi efetuada diariamente pesagem individual de cada saco com posterior abertura para avaliação do conteúdo e segregação de acordo com ANVISA, e nova pesagem dos resíduos corretamente segregados. Carcaças e cadáveres foram pesados separadamente.

\section{Resultados e Discussão}

Geração/ Segregação: Durante o período de estudo, na maior parte do hospital, foi observada apenas a segregação dos resíduos perfurocortantes. Apenas em dois laboratórios (Patologia Clínica e Parasitologia) os resíduos considerados recicláveis eram separados dos infectantes. Em nenhum ambiente avaliado ocorria a segregação conforme exigência da ANVISA. Segundo GARCIAe ZANETTI-RAMOS (2004), a segregação é o ponto fundamental de toda a discussão sobre a periculosidade ou não dos resíduos de serviços de saúde. Apenas uma parcela é potencialmente infectante, contudo, se ela não for segregada, todos os resíduos que a ela estiverem misturados também deverão ser tratados como potencialmente infectantes, exigindo procedimentos especiais para acondicionamento, coleta, transporte e disposição final, elevando assim os custos do tratamento desses resíduos. Acondicionamento: Em nenhuma área do hospital, com exceção do Bloco Cirúrgico, os recipientes de acondicionamento de RSS possuíam as características exigidas pela ANVISA (material lavável, resistente à punctura, ruptura e vazamento, com tampa provida de sistema de abertura sem contato manual, com cantos arredondados e resistentes ao tombamento). A exigência de sistema de abertura sem contato manual não se aplica aos recipientes de centros cirúrgicos, fato este que colaborou para a adequação deste local nas normas legais. Em laboratórios, salas de internamento e ambulatórios foram observadas caixas de papelão, caixas de madeira, baldes de plástico e latas de alumínio enferrujadas sendo utilizados como recipientes de 
acondicionamento. Identificação: Apenas as caixas para acondicionamento dos perfurocortantes e os sacos branco-leitosos estavam devidamente identificados conforme exigência legal. Nenhum recipiente de acondicionamento de RSS continha o símbolo de substância infectante - para grupo A, ou símbolo de risco associado, discriminação de substância química e frases risco - para grupo B (NBR 7500 da ABNT, 1987). Em maio de 2005 deuse início a coleta diferenciada dos RSS por empresa terceirizada, e passaram a ser utilizados recipientes para armazenamento intermediário (bombonas) que continham adesivos com símbolo e frase de risco. Coleta e transporte interno: A coleta interna era efetuada duas vezes ao dia, nos horários de 8:00hs e 16:00hs, por funcionárias de empresa terceirizada pela UFPR. Durante o acompanhamento desta etapa observou-se o uso de uniforme, luvas plásticas (algumas vezes dispensada) e touca. Não foi observado o uso de outro tipo de equipamento de segurança pessoal. SILVA (2004) em entrevista a funcionários de hospital do interior paulista constatou que $32,9 \%$ dos entrevistados já sofreram acidente durante manipulação de resíduos. Os perfurocortantes foram as maiores causas destes acidentes, totalizando $77,5 \%$. Dentre as medidas de prevenção de acidentes de trabalho consideradas importantes pelos funcionários, estavam: acondicionamento correto de perfurocortantes, manuseio cuidadoso de perfurocortantes, segregação de resíduos e uso de EPI, respectivamente. ANDRADE (1997), em trabalho realizado em 80 unidades hospitalares relatou que o uso de EPI não estava de acordo com as exigências da ABNT em nenhuma das unidades avaliadas, sendo luvas e uniformes os equipamentos utilizados na maioria dos casos. A falta de capacitação e desconhecimento da importância dos resíduos também demonstrou ser fator de risco para os manipuladores. Armazenamento intermediário: Para armazenamento intermediário era utilizada uma caixa d'água com capacidade para 1000 litros, em um corredor de baias de grandes ani- mais. A partir de maio de 2005 (coleta diferenciada) passaram a ser utilizadas bombonas plásticas para tal fim. Armazenamento externo: Para esta fase era utilizado um abrigo construído dentro do Setor de Ciências Agrárias, à aproximadamente 150 metros do Hospital, o qual era coberto, com muretas de alvenaria formando três divisões (resíduo comum, reciclável e de saúde) e telado em toda sua extensão. Os resíduos permaneciam neste abrigo até a coleta por caminhão da Prefeitura Municipal de Curitiba. Após o início da coleta diferenciada, o local de armazenamento externo passou a ser o mesmo de armazenamento intermediário (corredor de baias de grandes animais). Foi observada a ausência de uma sala de residuos, conforme exigência legal. Capacitação para manejo dos resíduos e estudos existentes. Nenhum estudo ou levantamento de RSS havia sido efetuado até o momento, na unidade hospitalar. Os funcionários da limpeza e servidores técnico-administrativos relataram que não haviam participado de nenhum tipo de curso de capacitação sobre manejo de RSS.

Quantificação dos resíduos gerados: PESAGEM 1 - Resíduos sólidos totais durante os 21 avaliados foram gerados $454,1 \mathrm{Kg}$ de resíduos segregados como RSS e 42,2 Kg de resíduos segregados como comuns. A média diária, no período estudado, foi de $21,62 \mathrm{Kg}$ para RSS e 2,01 Kg para o resíduo comum. Carcaças e cadáveres totalizaram 19,3 Kg. PESAGEM 2 - Resíduos perfurocortantes Durante os oito meses de avaliação foram produzidos $36,9 \mathrm{~kg}$ de perfurocortantes, correspondendo a uma média mensal de 4,61kg. PESAGEM 3 -Resíduos sólidos totais e posterior segregação $O$ gráfico a seguir (FIGURA 01) apresenta o resultado da pesagem, em primeira instância, com os resultados segundo a classificação e segregação realizada pelo hospital e, depois da análise, com a nova classificação, considerando as duas classes avaliadas -resíduos comuns e RSS. 
FIGURA 1 - QUANTIDADE TOTAL E MÉDIA DE RESÍDUOS GERADOS DE 4 DE MAIO A 2 DE JUNHO DE 2005, SEGUNDO SEGREGAÇÃO INTERNA DO HV-UFPR E SEGUNDO CLASSIFICAÇÃO PROPOSTA PELA ANVISA (CURITIBA, 2005).

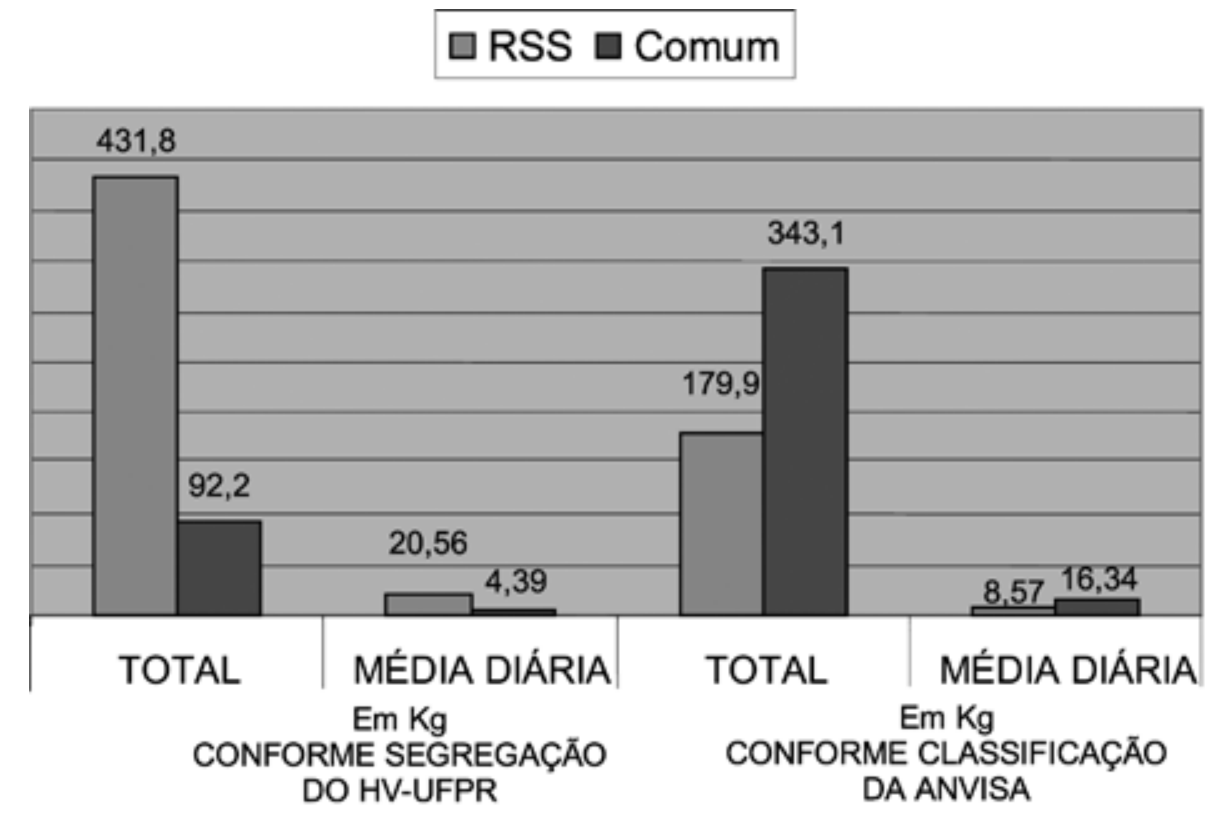

Os pesos carcaças e cadáveres foi de $427 \mathrm{Kg}$, sendo que deste total, $400 \mathrm{Kg}$ referem-se ao peso estimado de um eqüino que morreu durante o período de estudo.

Revelou-se a possibilidade de diminuição de $58,3 \%$ na quantidade de RSS apenas com a segregação, sendo que a média diária que era de $20,56 \mathrm{Kg}$ passou a ser de $8,57 \mathrm{Kg}$. Se considerada a recomendação do CONAMA de estimular a minimização de resíduos, promovendo a substituição de materiais e de processos por alternativas de menor risco, assim como a redução na fonte e a reciclagem, a diminuição na quantidade de resíduos sólidos poderia ser ainda maior.

\section{CONCLUSÕES}

Com este estudo foi possível chegar às seguintes conclusões: Nenhuma das etapas do gerenciamento interno de resíduos sólidos no hospital está de acordo com as exigências legais em vigor; A segregação dos resíduos negligencia aspectos importantes do gerenciamento de RSS (como minimização) e provoca um aumento significativo na quantidade total de resíduos segregados como RSS; A diminuição na quantidade total de RSS gerada poderia chegar a $58 \%$ caso seja feita uma segregação conforme determinam a ANVISA e o CONAMA; Não existem dados suficientes sobre RSS gerados em ambientes veterinários, e não existem números oficiais que indiquem a quantidade gerada nestes estabelecimentos.

\begin{abstract}
ANDRADE, J. B. L. Análise do Fluxo e das Características Físicas, Químicas e Microbiológicas dos Resíduos de Serviços de Saúde: Proposta de Metodologia para o Gerenciamento em Unidades Hospitalares. Tese (Doutorado). Escola de Engenharia de São Carlos: Universidade de São Paulo, 1997.
\end{abstract}

ARMOND, G. A.; AMARAL. A. F. H. In: Manual de Infecção Hospitalar: Epidemiologia, Prevenção e Controle. Gerenciamento de Resíduos de Serviços de Saúde (Lixo Hospitalar). Comissão de Controle de Infecção Hospitalar (CCIH). MEDSI. Hospital das Clínicas da UFMG, cap. 54, p.734-742. Rio de Janeiro, 2001.

ASSOCIAÇÃO BRASILEIRA DE NORMAS TÉCNICAS. NBR 7500. Símbolos de Risco e Manuseio para o Transporte Rodoviário de Produtos Perigosos. Rio de Janeiro:ABNT, 1987.

BRASIL. Agência Nacional de Vigilância Sanitária. RDC $n^{\circ}$ 306. Dispõe sobre o Regulamento Técnico para o Gerenciamento dos Resíduos de Serviços de Saúde. ANVISA, 2004. 
Conselho Nacional de Meio Ambiente. Resolução $n^{\circ} 358$. Dispõe sobre o tratamento e a destinação final dos resíduos de serviços de saúde e dá outras providências. CONAMA, 2005.

FERREIRA, J. A. Resíduos Sólidos e Lixo Hospitalar: Uma Discusão Ética. Cadernos de Saúde Pública, v. 11,n.2, p.314-320, 1995.

GARCIA, L. P.; ZANETTI-RAMOS, B. G. Gerenciamento dos resíduos de serviços de saúde: uma questão de biossegurança. Cadernos de Saúde Pública, v. 20, n.3, p. 744-752, 2004.

KRAUSS, A.J. Waste management: Small Animal Practice. Journal of the American Veterinary Medical Association, v. 223, n.1, p. 46-47, 2003.

SILVA. M. F. I. Resíduos de Serviços de Saúde Gerenciamento no Centro Cirúrgico, Central de Material e Centro de Recuperação Anestésica de um Hospital do Interior Paulista. Tese (Doutorado). Escola de Enfermagem de Ribeirão Preto: Universidade de São Paulo, 2004.
SUBERKROPP, K.F.; KLUG, M.J. Microbiology ecology. v.1, p. 96-123, 1974. apud ARMOND, G. A.; AMARAL. A. F. H. In: Manual de Infecção Hospitalar: Epidemiologia, Prevenção e Controle. Gerenciamento de Resíduos de Serviços de Saúde (Lixo Hospitalar). Comissão de Controle de Infecção Hospitalar (CCIH). MEDSI. Hospital das Clínicas da UFMG, cap. 54, p.734-742, 2001.

VOLKOW, P. Contact with hospital syringes containing body fluids. Implications for medical waste management regulation. Salud Publica de México, v. 45, n. 2 , p. 15-25, 2003.

Recebido para publicação:

$15 / 05 / 2007$

Aprovado:

$13 / 01 / 2008$ 